\title{
Laser Assisted Endoscopic Stapes Surgery: Single Operator Outcomes
}

\author{
Ryan Chin Taw Cheong ${ }^{1}$, Bik Kotecha ${ }^{1,2}$, Gaurav Kumar ${ }^{1}$ \\ ${ }^{1}$ Barking, Havering and Redbridge University Hospitals NHS Trust, ${ }^{2}$ Royal \\ National Throat, Nose and Ear Hospital
}

\section{Introduction}

Noting that the microscope is limited by direct line of sight during procedures, several intraoperative advantages have been identified since Tarabichi described the first endoscopic stapes procedure in 1999 (1). The authors provide a summary of endoscopic stapes surgery highlighting the audiometric and patient outcomes.

\section{Methods}

Retrospective analysis between May 2017 and September 2017 of 7 patients who underwent laser assisted endoscopic stapedotomy or stapedectomy extracting pre-determined audiometric and patient outcomes with subsequent data processing via Microsoft Excel.

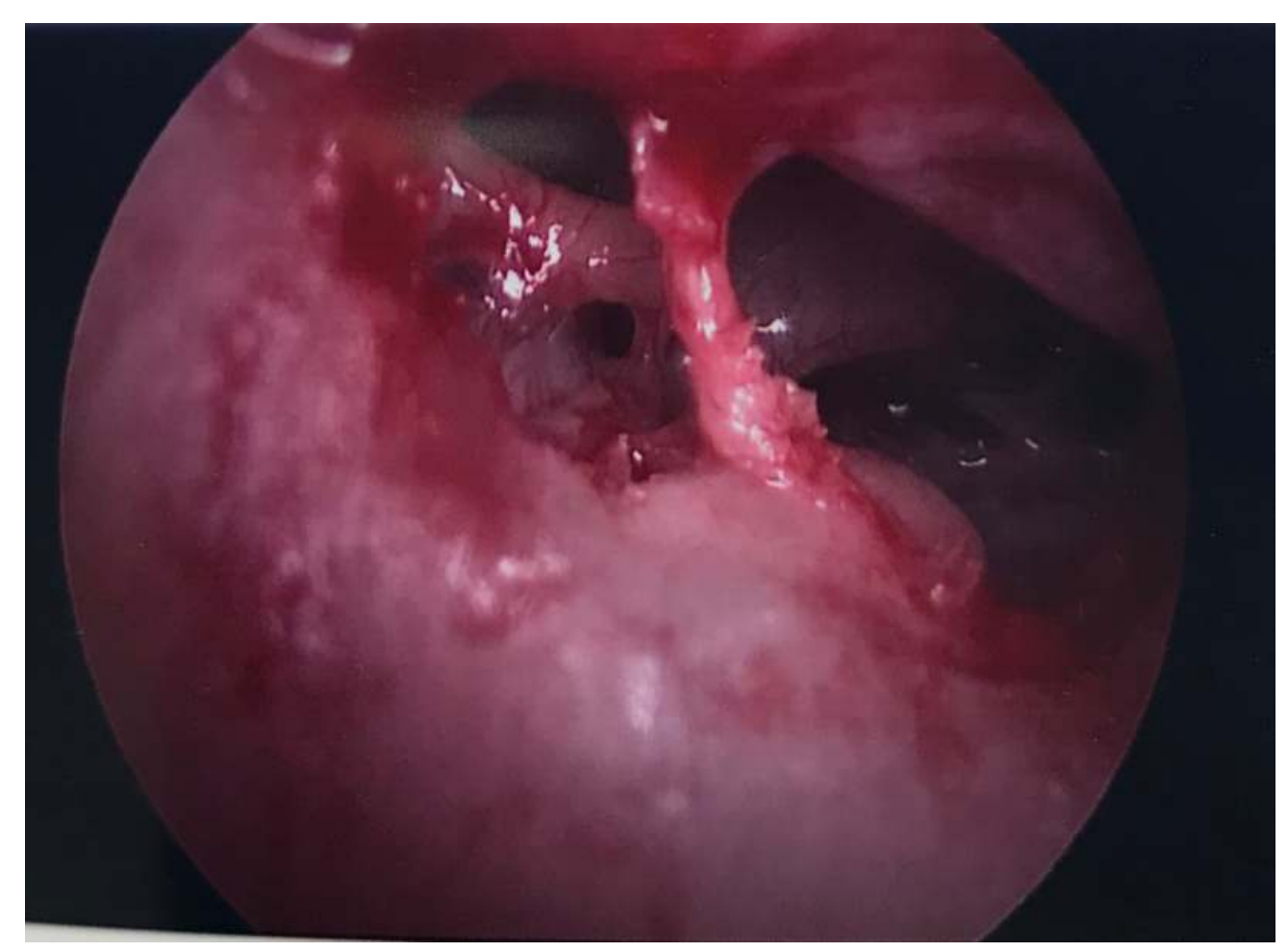

\section{Results}

The mean follow-up period was 10 weeks with up to $71.4 \%$ of patients achieving a post-operative air-bone gap closure of less than $10 \mathrm{~dB}$. This is comparable to the audiometric outcomes reported in the literature between $56.0 \%$ to $86.7 \%$ (2). The mean pre-operative air-bone gap was $40 \mathrm{~dB}$ and mean post-operative airbone gap was $9 \mathrm{~dB}$. There were no reports of postoperative dysgeusia with $100 \%$ preservation of the chorda tympani.

\section{Discussion}

Proponents believe that the endoscope improves visualization during stapes surgery. Specifically, the endoscope's wide angle viewing, improved visualization of parallel structures, and line of sight closer to the surgical field have been cited as factors that can potentially lead to improved outcomes and more effective instructional aides.

Generally speaking, side by side comparisons between endoscopic and microscopic stapes surgery report that there are no differences in hearing outcomes or complication rates.

Although many endoscopic surgeons quote reduced rates of chorda tympani manipulation, the authors reported that the chorda tympani nerve was still manipulated in $92.2 \%$ of cases.

Although there were some complications intraoperatively, including $7.8 \%$ of patients with tympanic membrane perforations, $3.9 \%$ with floating footplates, and1patient whose incus subluxed,no patient reported any post-operative complication.

Regarding audiometric outcomes, the median postoperative air-bone gap was $9.0 \mathrm{~dB} \mathrm{HL}$, with $90.2 \%$ of patients closing their air-bone gap to less than $20 \mathrm{~dB}$ HL (3).

\section{Conclusion}

Endoscopic stapes surgery is an effective alternative when compared to microscopic stapes surgery, with similar complication rates and audiometric outcomes.

1. Tarabichi M. Endoscopic middle ear surgery. Annals of Otology, Rhinology \& Laryngology. 1999 Jan;108(1):39-46.

2. Hunter JB, Rivas A. Outcomes following endoscopic stapes surgery. Otolaryngologic Clinics of North America. 2016 Oct 1;49(5): 1215-25.

3. Hunter JB, Zuniga MG, Leite J, et al: Surgical and audiologic outcomes in endoscopic stapes surgery across 4 institutions. Otolaryngol Head Neck Surg 54(6):1093-1098, 20 